\section{Response to Comment by D.C.F. Fayle}

Let me first say that I am pleased that Mr. Fayle found my short article interesting enough to stimulate him to write about it. His primary criticism, I believe, is that linear regression is not the best vehicle to describe relative growth roles. $\mathrm{He}$ is, of course, correct. I recognized this deficiency and plotted the data on the graphs to more clearly show the growth trends. His graphs simply show the data in a different way.
Although the current annual height curves cross at approximately age 40 on both sites, this is most likely a result of significant competition retarding the growth of the larch. What the relative growth rates might be under more controlled conditions is open to conjecture. Under proper management and thinning regimes, I would not expect the reduction in height and diameter growth that occurred under natural conditions.

I continue to believe that the importance and stature of larch will increase in the future.

Douglas R. Mead

\title{
The CIF Scene
}

\section{New Members}

\section{Active}

\section{E. E. Cloney}

1890 Spruce St. Apt \#104

Prince George, B.C.

W. T. Davis

Alberta Forest Service

Box 3123

Edson, Alta. TOE OPO

T. H. Hall

P.F.R.C. 506 W. Burnside Rd.

Victoria, B.C. V8Z $1 \mathrm{M} 5$

\section{Gendreau}

Centre Res. For. Laurentides

C.P. 3800

Ste Foy, P.Q. G1V 4C7

G. D. Lloyd

\#2 - 964 10th Ave. E.,

Prince Rupert, B.C.

\section{L. McCormack}

22 Myrtie St.,

Orono, Maine 04469 USA

R. J. O'Connell

c/o Maritime For. Ranger School

R R \#5

Fredericton, N.B. E3B 4X6

R. W. Parker

5.9015 95 Ave.,

Edmonton, Alta. T6C 1 Z2

W. J. Pearson

2402 Marlborough Dr.,

Nanaimo, B.C. V9S 3J5

\section{S. J. Pedersen}

P.O. Box 24

Holberg, B.C. VON 1 ZO

R. A. Sexsmith

P.O. Box 1720

Prince Albert, Sask. S6U 5T3

G. V. Skabeikis

P.O. Box 89

Holberg, B.C. VON 1 ZO
S. M. Smith

Box 17 Site 18 RR\#5

Prince Albert, Sask. S6V 5R4

J. H. Spencer

Box 10

Meadow Lake, Sask. SOM 1VO

J. H. Stephenson

\#6-10554 - 85 Ave.,

Edmonton, Alta. T6E 2K4

D. C. Trudeau

492 Pembroke St. E. Apt. \#4

Pembroke, Ont.

\section{Student}

B. D. Byford

166 Beverly Court

Ancaster, Ont. L9G 1C1

W. B. Clark, Jr.

302 Aroostook Hall

Univ. Maine

Orono, Maine 04473, USA

F. J. Conlon, Jr.

111 Nutting

Orono, Maine 04469, USA

P. J. Cushman

403 Estabrooke Hall

Univ. Maine

Orono, Maine 04469, USA

Julie I. Eldridge

2544 Eldridge Rd.

E. Aurora, N.Y. USA

C. J. Gadzik

Box 12

Milford, Maine 04461, USA

M. Gendrau

2725 rue Restigouche

Ste-Foy, P.Q. G1V 1E3

B. D. Harvey

clo Dept. Forest Resources

UNB - Fredericton, N.B.

E3B 4J1
C. Howard

11 Third Ave.,

Orangeville, Ont. L9W 1 G8

G. F. Leslie

55 Glengarry Road,

Brockville, Ont. K6V 2N5

P. K. Love

352 W. Hart Cres.

Burlington, Ont. L7N 1N7

Karen Mikoliew

Box 1720

Prince Albert, Sask.

S6V 5T3

K. G. Moar

Box 89

Montague, P.E.I. COA 1R0

T. C. Rankin

8943 Baylor Cres. S.W.

Calgary, Alta. T2V 3N5

C. Stamplecoskie

271 Sixth St.

Toronto, Ont. M8V 3 A8

P. J. Strauch

765 South Main St.,

Old Town, Maine 04468, USA

B. Voldock

Box 548

Barry's Bay, Ont. KOJ 1B0

\begin{tabular}{|lr|}
\hline \multicolumn{3}{|c|}{ Current CIF Membership } \\
\hline Honorary $\ldots \ldots \ldots \ldots \ldots \ldots \ldots \ldots$ & 2 \\
Fellow $\ldots \ldots \ldots \ldots \ldots \ldots \ldots \ldots$ & 26 \\
Active $\ldots \ldots \ldots \ldots \ldots \ldots \ldots \ldots$ & 1844 \\
Affiliate $\ldots \ldots \ldots \ldots \ldots \ldots \ldots \ldots$ & 91 \\
Student $\ldots \ldots \ldots \ldots \ldots \ldots \ldots \ldots$ & $\begin{array}{r}942 \\
\text { Retired } \ldots \ldots \ldots \ldots \ldots \ldots \ldots\end{array}$ \\
Temporarily Lost $\ldots \ldots \ldots \ldots \ldots$ & 164 \\
\hline Total: $\ldots \ldots \ldots \ldots \ldots \ldots \ldots \ldots$ & 48 \\
\hline
\end{tabular}

